\title{
Endocarditis infecciosa asociada a catéter de hemodiálisis: hallazgos clínicos y ecocardiográficos
}

\author{
Luis Ediap $^{1-3}$, Samuel Córdova ${ }^{2}$, Eric Zúñiga ${ }^{1-3}$, Pedro Usedo $^{1}$, Alex Muñoz $^{3}$, Gabriela Fernández ${ }^{3}$, Fabiola Reales ${ }^{3}$, Marinka Gladic $^{3}$. \\ 1. Clínica Antofagasta, Universidad de Antofagasta, Chile. \\ 2. Hospital Clínico Universidad Católica, Santiago, Chile. \\ 3. Facultad de Medicina y Odontología, Universidad de Antofagasta, Chile.
}

Hallazgos clínicos y ecocardiográficos en pacientes con endocarditis infecciosa (E.I.) asociada a catéter de hemodiálisis (CHD) fueron comparados con sujetos controles con E.I. "habitual".

Pacientes y métodos: Del total de registros ecocardiográficos de una Clínica privada de Antofagasta entre 2009 y 2014, 4 pacientes presentaban E.I. asociada CHD (E.I.-CHD) y 16 otro tipo de E.I. (controles). Se compararon ambos grupos clínicamente y en las distintas modalidades de ecografía, para evaluar el aporte de cada una de ellas en el diagnóstico, manejo y comprensión del mecanismo de producción de la E.I.

Resultados: El periodo del inicio de los síntomas hasta la hospitalización y el comprendido entre ésta hasta el fallecimiento fue variable, con tendencia a fallecimiento más precoz en el grupo E.I.-CHD (promedio 14,6 vs 20,6 días en el grupo control). La edad en E.I.-CHD fue mayor (65 versus 62.2 años), con mayor incidencia de las 3 comorbilidades más frecuentes: hipertensión arterial (100\% versus 56,25\%), diabetes mellitus (75\% versus $50 \%)$ e insuficiencia cardíaca $(75 \%$ versus $31,25 \%$ ). La mortalidad fue $18.75 \%$ en el grupo control y $75 \%$ en la E.I.-CHD. En los pacientes con E.I.-CHD se aisló: Cándida (50\%), Bacilos gram nega- tivo (Stenotrophomonas maltophilia) (25\%) y SAMR (25\%) mientras que en el grupo control fueron aislados Enterococcus faecalis (25\%), Streptococcus viridans (12.5\%), Streptococcus spp (6.25\%), Staphylococcus aureus $(6.25 \%)$, Streptococcus grupo C (6.25\%), Abiotrophia defectiva $(6.25 \%)$ y Streptococcus pneumoniae (6.25). En $31.25 \%$ de los casos los hemocultivos fueron negativos. La localización más frecuente de las vegetaciones en los pacientes con E.I.-CHD correspondió a la pared de $\mathrm{AD}$, sitio de impacto del jet del CHD, en un $100 \%$, seguida por CHD $50 \%$, tricúspide $25 \%$ y aórtica $25 \%$. En los controles las localizaciones fueron $75 \%$ en válvula aórtica y $25 \%$ en válvula mitral. En el grupo de E.I.-CHD, la mitad presentó disfunción sistólica e insuficiencia valvular, mientras que en el grupo control fue predominante la insuficiencia valvular $(62,5 \%)$ seguida por la perforación de velo $(25 \%)$.

Conclusión: En las E.I.-CHD se aislaron gérmenes asociados a un peor pronóstico (hongos o bacterias atípicas), con altísima mortalidad. La localización principal de las vegetaciones fue en la pared de la aurícula derecha (AD), alrededor de la desembocadura de la vena cava inferior (VCI), sitio de impacto del jet del $\mathrm{CHD}$, y en el tercio distal del catéter. 


\section{Infective endocarditis associated with hemodialysis catheters: clinical and ehocardiographic findings}

Clinical and echocardiographic findings in patients with infective endocarditis (I.E.) associated to the presence of hemodialysis catheters (HDC) were compared to those in subjects with other types of I.E.

Methods: Between 2009 and 2014 an echocardiographic diagnosis of I.E. was established in 4 subjects with a hemodialysis catheter in place and 16 patients had a common variety of I.E. (controls). Clinical and echocardiographic findings, including echocardiographic modalities were compared between groups.

Results: Time from onset of symptoms and time to patient death were shorter in I.E.-HDC patients compared to controls (mean 14.6 vs 20.6 days, respectively. I.E.-HDC patients tended to be older $(65.0$ vs 62.2 years old). The incidence of main co-morbidities was higher in I.E.-HDC: hypertension $(100 \%$ vs $56.2 \%$ ), diabetes (75 vs $59 \%$ ) and heart failure ( $75 \%$ vs $31.2 \%$ ), respectively. Mortality was much higher in I.E-HDC (74\% vs 18\%). Infective agents also differed between groups: I.E.-HDC was associate to $\mathrm{C}$ albicans (50\%), Gram negative rods (Stenotrophomonas maltophilia) (25\%) y SAMR (25\%). In contrast, the agents involved in controls were $\mathrm{E}$ faecalis (25\%), S viridans (12.5\%), S. group C (6.25\%), $\mathrm{S}$ aureus $(6,25 \%)$, group C Streptococcus $(6.25 \%)$, Abiotrophic defectiva (6.25\%) and S pneumoniae $(6.25 \%)$. Overall, blood cultures were negative in $31.2 \%$ patients. Among patients with I.E.-HDC vegetations were most commonly found at the right atrial wall $(100 \%)$, on the catheter $(50 \%)$, at the tricuspid valve (25\%) and at the aortic valve (25\%). In non I.E.-HDC, vegetations were located at the aortic valve $(75 \%)$ and the mitral valve $(25 \%)$. Half the patients with I.E.-HDC patients presented ventricular dysfunction and valvular regurgitation while control patients had predominantly valvular insufficiency (62.5\%) or leaflet perforation (25\%).

Conclusion: Mortality in I.E.-HDC patients was very high, associated to the presence of aggressive microbial or fungal agents. Vegetations were most commonly located at the right atrial wall, around de junction with the inferior vena cava and on the catheter itself.

Key Words: endocarditis, renal dialysis, catheters, echocardiography. 


\section{Inroducción:}

La E.I.-CHD reúne características especiales, como la permanencia del catéter en su sitio un tiempo prolongado, la necesidad de que éste se ubique en la AD para proporcionar un flujo adecuado y alto (200 a $450 \mathrm{ml} /$ $\min ^{5-9}$ ) para la diálisis, lo cual produce un jet de alta velocidad, llevando a una lesión en el sitio del impacto, aumentando la posibilidad de trombosis y su posterior colonización. Estos pacientes presentan altas comorbilidades, inmunocompromiso y una ruptura permanente de la barrera mecánica de la piel por la presencia del catéter. El valor de la ecocardiografía en todas sus modalidades es de incuestionable valor en la E.I., tanto diagnóstico, como para la detección de complicaciones y toma de decisiones terapéuticas. La ETE 2D y, actualmente, la 3D proporcionan una mayor sensibilidad y especificidad en el diagnóstico y detección de complicaciones, sobre todo en las cavidades izquierdas. Para el diagnóstico de vegetaciones, la ETT tiene una sensibilidad de $75 \%$ y especificidad de $90 \%$ pudiendo ser menor en casos de ventanas acústicas subóptimas, vegetaciones pequeñas, portadores de prótesis valvulares o dispositivos intracardiacos. En tanto, la ETE alcanza sensibilidades entre 85 y $90 \%$ y una especificidad mayor del $90 \% 12$. La ETT permite valorar la válvula tricúspide con bastante precisión, pudiendo visualizar las vegetaciones en la mayoría de los casos. Sin embargo, vegetaciones en cable de marcapaso, catéteres y prótesis del lado derecho son particularmente difíciles de diagnosticar porque las reverberaciones y los artefactos producidos por el material intracardiaco pueden enmascarar pequeñas vegetaciones asociadas a estas estructuras. La ETE estaría indicada en caso de ventanas acústicas subóptimas o alta sospecha clínica con ETT negativo. Además, es más sensible para detectar signos de endocarditis en válvula pulmonar o vegetaciones ancladas a la válvula de Eustaquio, la red de Chiari, o a catéteres o dispositivos intravasculares. A pesar de la superioridad de la ETE, la ETT siempre es obligatoria como examen inicial. El objetivo del estudio es investigar si existen diferencias entre una endocarditis "habitual" y una asociada a catéter de hemodiálisis. Para lograr este objetivo se diseñó un grupo control similar en todas las otras características (ej: misma institución, esquema de tratamiento, grupo de médicos tratantes, periodo de estudio, etc.); se comparó los hallazgos clínicos, ecocardiográficos y la utilidad de las diferentes técnicas de Ecocardiografía para evaluar la E.I.-CHD, considerando la siguiente información: diagnóstico clínico y ecográfico, característica de la vegetación, zona afectada y/o presencia de insuficiencia valvular, tipo y tiempo de permanencia del catéter, comorbilidades, hemocultivos, microorganismo y sensibilidad de éste, tipo y duración de tratamiento antibiótico y, finalmente, evolución y pronóstico.

\section{Material y Método:}

Se realizó un estudio retrospectivo de la totalidad de 10.633 registros ecocardiográficos realizados en la Clínica Antofagasta de la ciudad de Antofagasta, Chile, entre Enero de 2009 y Julio de 2014. Durante este periodo se utilizaron 3 equipos: ecógrafo ATL-HDI 5000 transductores ETT P4-2, ETE MPT 7-4, Philips iE33 transductores ETT X 5-1, ETE X7-2T y GE Vivid E9 transductores ETT M5S-D, ETE 6VT-D. Del registro de ecocardiografías realizadas se preseleccionaron los pacientes que presentaban los siguientes criterios: control ecográfico de E.I., antecedente de E.I. tratada, síndrome febril como motivo de consulta y auscultación de un soplo cardíaco. Del universo de 10.633 ecocardiogramas se seleccionaron 20 pacientes que cumplían con los criterios mencionados y, además, con los criterios de Duke modificados para E.I. ${ }^{15}$. Estos 20 pacientes fueron separados en dos grupos: el grupo en estudio, el cual presentó E.I.-CHD; y el grupo control, no asociado a CHD. Cada grupo cumple los siguientes criterios: E.I.-CHD: E.I. confirmada según criterios de Duke con ecocardiograma positivo, más las siguientes características: paciente con enfermedad renal crónica en etapa V en HD, dializado mediante CHD (independiente del tiempo de uso) y hemocultivo positivo, tanto en CHD como en hemocultivo periférico; y Grupo control: E.I. confirmada según criterios de Duke con ecocardiograma positivo y no estar sometido a HD. Se compararon ambos grupos enfocándose en la búsqueda de la siguiente información: tipo de ecógrafo utilizado, técnica utilizada, rendimiento de ésta, diagnóstico clínico y ecográfico; característica de la vegetación, zona afectada y/o presencia de insuficiencia valvular, tipo de catéter y tiempo de permanencia, hallazgos clínicos, comorbilidades, hemocultivos, microorganismo y sensibilidad, tipo y duración de tratamiento antibiótico, y evolución (Tablas 1 y 2). Del grupo de E.I. -CHD, correspondiente a un total de 4 pacientes, se realizó un estudio detallado de su historial clínico y ecográfico (Tabla 3), además de sus imágenes ecocardiográficas.

Análisis estadístico: Los resultados del análisis descriptivo se presentan en valores absolutos seguidos del porcentaje. 


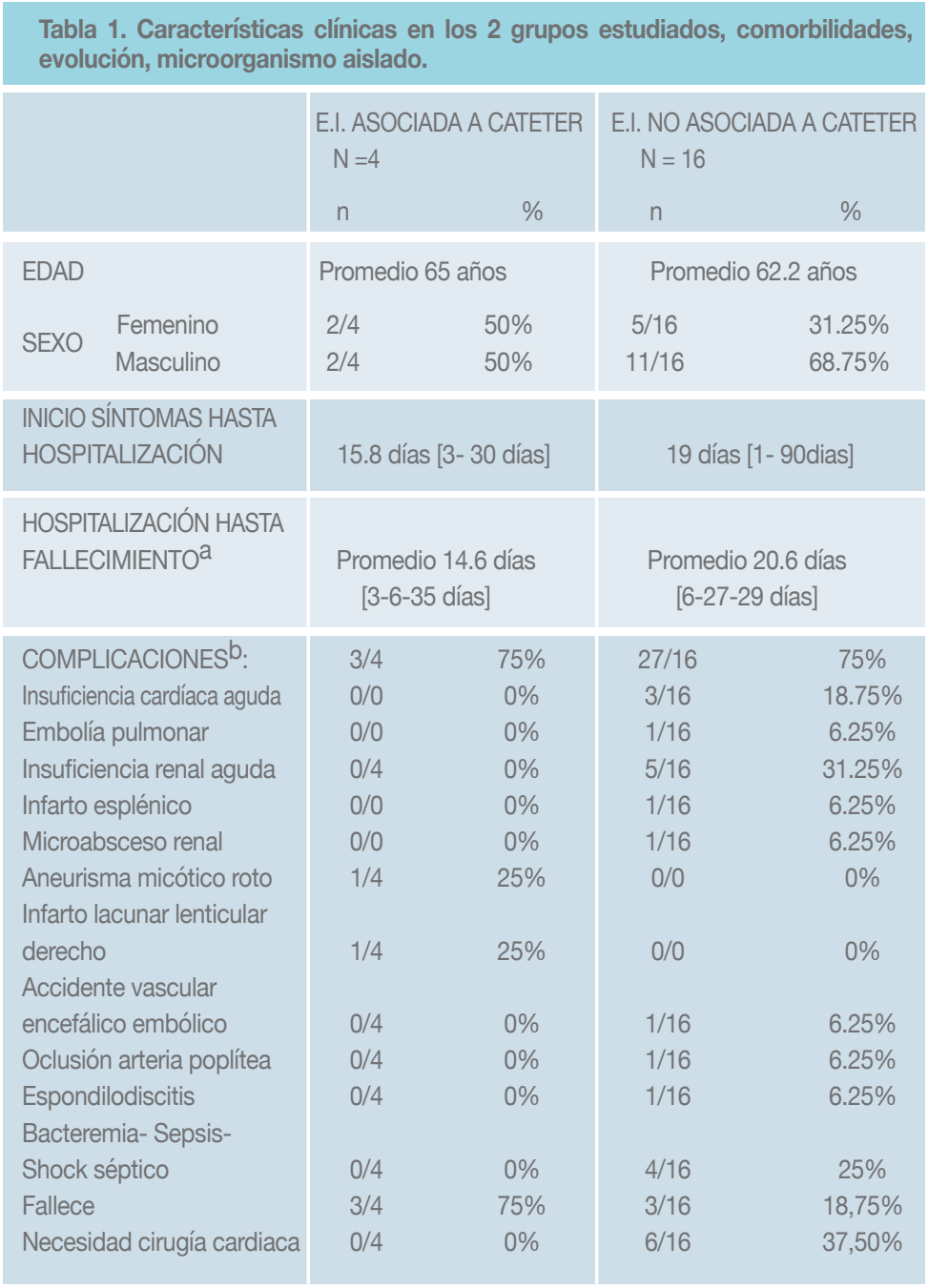

a Los números entre corchetes corresponde a los días desde la hospitalización hasta el fallecimiento de cada paciente.

b Complicaciones: presenta mayor $\boldsymbol{n}$ debido a que algunos pacientes presentaron más de una complicación.

Tabla 2. Hallazgos microbiológicos, en la E.I. asociada a catéter y la no asociada a catéter

\begin{tabular}{l|c|c}
\hline & E.I. Asociada a Catéter & E.I. No asociada a catéter \\
\hline SAMR & 1 & 0 \\
Candida spp & 1 & 0 \\
Candida famata & 1 & 0 \\
Stenotrophomonas maltophilia & 1 & 0 \\
Streptococcus viridans & 0 & 2 \\
Streptococcus spp & 0 & 1 \\
SAMS & 0 & 1 \\
Enterococcus faecalis & 0 & 4 \\
Abiotrophia defectiva & 0 & 1 \\
Streptococcus Grupo C & 0 & 1 \\
Streptococcus pneumoniae & 0 & 1 \\
Desconocido & 0 & 5 \\
Total & 4 & 16
\end{tabular}




\begin{tabular}{|c|c|c|c|c|}
\hline & \multicolumn{2}{|c|}{$\begin{array}{l}\text { E.I. ASOCIADO A } \\
\text { CATETER } \\
\mathrm{N}^{\circ} \text { CASOS }=4\end{array}$} & \multicolumn{2}{|c|}{$\begin{array}{l}\text { E.I. NO ASOCIADO } \\
\text { A CATETER } \\
N^{\circ} \text { CASOS }=16\end{array}$} \\
\hline & $\mathrm{n}$ & $\%$ & $\mathrm{n}$ & $\%$ \\
\hline \multicolumn{5}{|l|}{ TIPO DE ECOCARDIOGRAFIA } \\
\hline ETT & $4 / 4$ & $100 \%$ & $16 / 16$ & $100 \%$ \\
\hline ETE & $1 / 4$ & $25 \%$ & $12 / 16$ & $75 \%$ \\
\hline \multicolumn{5}{|l|}{ INSUFICIENCIA VALVULAR } \\
\hline $\begin{array}{l}\text { MODERADA-SEVERA EN VALVULA CON E.I. } \\
\text { INSUFICIENCIA VALVULAR }\end{array}$ & $2 / 4$ & $50 \%$ & $10 / 16$ & $62.5 \%$ \\
\hline \multicolumn{5}{|l|}{ VEGETACIONES ${ }^{a}$} \\
\hline Válvula Aórtica nativa & $1 / 4$ & $25 \%$ & $8 / 16$ & $50 \%$ \\
\hline Válvula Mitral nativa & $0 / 4$ & $0 \%$ & $4 / 16$ & $25 \%$ \\
\hline Válvula Tricúspide & $1 / 4$ & $25 \%$ & $0 / 16$ & $0 \%$ \\
\hline Pared Aurícula Derecha & $4 / 4$ & $100 \%$ & $0 / 16$ & $0 \%$ \\
\hline Válvula Aórtica Protésica & $0 / 4$ & $0 \%$ & $3 / 16$ & $18,7 \%$ \\
\hline Válvula Mitral Protésica & $0 / 4$ & $0 \%$ & $1 / 16$ & $6.2 \%$ \\
\hline Catéter & $2 / 4$ & $50 \%$ & $0 / 16$ & $0 \%$ \\
\hline Cable Marcapaso & $0 / 4$ & $0 \%$ & $2 / 16$ & $12,5 \%$ \\
\hline Tracto de salida de Ventrículo izquierdo & $0 / 4$ & $0 \%$ & $2 / 16$ & $12,5 \%$ \\
\hline HALLAZGOS ECOGRÁFICOSb & $4 / 4$ & & $26 / 16$ & \\
\hline Disfunción sistólica (FE<50\%) & $2 / 4$ & $50 \%$ & $3 / 16$ & $18,7 \%$ \\
\hline Abscesos $^{c}$ & $0 / 0$ & $0 \%$ & $3 / 16$ & $18,7 \%$ \\
\hline Perforación de velo ${ }^{d}$ & $0 / 0$ & $0 \%$ & $4 / 16$ & $25 \%$ \\
\hline \multicolumn{5}{|l|}{ Insuficiencia valvular } \\
\hline - Aórtica & $1 / 4$ & $25 \%$ & $7 / 16$ & $43,7 \%$ \\
\hline - Mitral & $0 / 4$ & $0 \%$ & $2 / 16$ & $12,5 \%$ \\
\hline - Mitro- aórtica & $0 / 4$ & $0 \%$ & $1 / 16$ & $6,2 \%$ \\
\hline -Tricuspídea & $1 / 4$ & $25 \%$ & $0 / 16$ & $0 \%$ \\
\hline Prolapso válvula mitral & $0 / 0$ & $0 \%$ & $1 / 16$ & $6,2 \%$ \\
\hline Ruptura cuerdas tendíneas & $0 / 0$ & $0 \%$ & $1 / 16$ & $6,2 \%$ \\
\hline Aquinesia & $0 / 0$ & $0 \%$ & $1 / 16$ & $6,2 \%$ \\
\hline Hipertensión pulmonar & $0 / 0$ & $0 \%$ & $2 / 16$ & $12,5 \%$ \\
\hline Derrame pericárdico & $0 / 0$ & $0 \%$ & $1 / 16$ & $6,2 \%$ \\
\hline
\end{tabular}

a. Vegetaciones: el aumento del n es debido a que varios pacientes presentaron más de un sitio con vegetación en ambos grupos estudiados.

b. Hallazgos ecográficos: en los 16 pacientes del grupo de no asociados a catéter se presentaron 26 complicaciones.

c. Abscesos incluyen las siguientes localizaciones: Tracto de salida de ventrículo izquierdo, anillo á́rtico y fibrosa

mitro- aórtica.

d. Perforación de velo incluye las siguientes localizaciones: no coronario y de válvula mitral.

\section{Resultados:}

Dentro de los resultados comparativos se observó que entre el periodo del inicio de los síntomas hasta la hospitalización y el comprendido entre ésta hasta el fallecimiento es bastante variable (Tabla 1), pero con tendencia a un fallecimiento más precoz en el grupo E.I.-CHD (promedio 14,6 días vs 20,6 días en el grupo control). Además, el grupo de E.I.-CHD presenta pacientes levemente más añosos (65 versus 62.2 años en los controles), con mayor incidencia de las 3 comorbilidades más detectadas en ambos grupos (Figura 1), a saber hipertensión arterial (100\% versus $56,25 \%$ ), diabetes mellitus ( $75 \%$ versus $50 \%$ ) e insuficiencia cardíaca ( $75 \%$ versus $31,25 \%$ ). La mortalidad en nuestro estudio fue $18.75 \%$ en el grupo control, mientras que en los casos de E.I.-CHD esta fue $75 \%$. Los gérmenes aislados fueron claramente diferentes en am- 
Tabla 4. Características ecográfica y clínica de cada uno de los pacientes del grupo de E.I. asociado a catéter de HD

\begin{tabular}{|c|c|c|c|c|}
\hline & CASO 1 & CASO 2 & CASO 3 & CASO 4 \\
\hline EDAD & 70 años & 72 años & 65 años & 53 años \\
\hline ETT2D permitió hacer el diagnóstico & Sí & Sí & Sí & No \\
\hline ETE 2D y 3D permitió hacer el diagnóstico ${ }^{a}$ & - & - & - & Sí \\
\hline Número de vegetaciones por ETT 2D & 3 & 2 & 2 & 1 \\
\hline Número de vegetaciones por ETE $2 \mathrm{D}$ y $3 \mathrm{D}$ & - & - & - & 2 \\
\hline Dimensión de la vegetación de mayor tamaño por ETT 2D & $3,1 \mathrm{~cm}$ & $1,6 \mathrm{~cm}$ & $2,4 \mathrm{~cm}$ & $1,8 \mathrm{~cm}$ \\
\hline Dimensión de la vegetación de mayor tamaño por ETE 2D y 3D & - & - & - & $2 \mathrm{~cm}$ \\
\hline Visualización vegetación en catéter HD por ETT & No & Sí & No & No \\
\hline Visualización vegetación en catéter de HD por ETE 2D y 3D & - & - & - & Sí \\
\hline Localización vegetación por ETT 2D & $\mathrm{AD} / \mathrm{T}$ & $\mathrm{AD} / \mathrm{CT}$ & ADNAo & $A D ?$ \\
\hline Localización vegetación por ETE 2D y 3D & - & - & - & $\mathrm{AD} / \mathrm{CT}$ \\
\hline $\mathrm{FE}<50 \%$ & No & Sí & Sí & No \\
\hline Hipertrofia ventricular izquierda & Sí & Sí & Sí & Sí \\
\hline Dilatación ventricular derecha & Sí & No & No & No \\
\hline Insuficiencia valvular moderada a severa & Tricúspide & Mitral & Aórtica & No \\
\hline Número de comorbilidades presentes & 4 & 4 & 3 & 3 \\
\hline Gérmen productor E.I. ${ }^{\mathrm{C}}$ & SAMR & Stenotrophomonas maltophilia & Cándida sp & Cándida famata \\
\hline Fallece & Sí & Sí & Sí & No \\
\hline
\end{tabular}

a. No se realizó ETE.

b. AD: aurícula derecha, VT: válvula tricúspide, CT: catéter de HD, VAo: velos aórticos.

c.SAMR, Staphylococcus aureus meticilino resistente.

bos grupos; en los pacientes con E.I.-CHD se aislaron Cándida (50\%), Bacilos Gram negativo (Stenotrophomonas maltophilia) (25\%) y SAMR (25\%). En cambio, en el grupo control fueron aislados: Enterococcus faecalis (25\%), Streptococcus viridans (12.5\%), Streptococcus spp (6.25\%), Staphylococcus aureus (6.25\%), Streptococcus grupo C (6.25\%), Abiotrophia defectiva (6.25\%) y Streptococcus pneumoniae $(6.25 \%)$, predominando claramente los Gram positivos con $68.75 \%$. Sin embargo, en $31.25 \%$ de los casos se obtuvieron hemocultivos

Figura 1. Características clínicas en los 2 grupos estudiados, comorbilidades, evolución y microorganismo aislado.

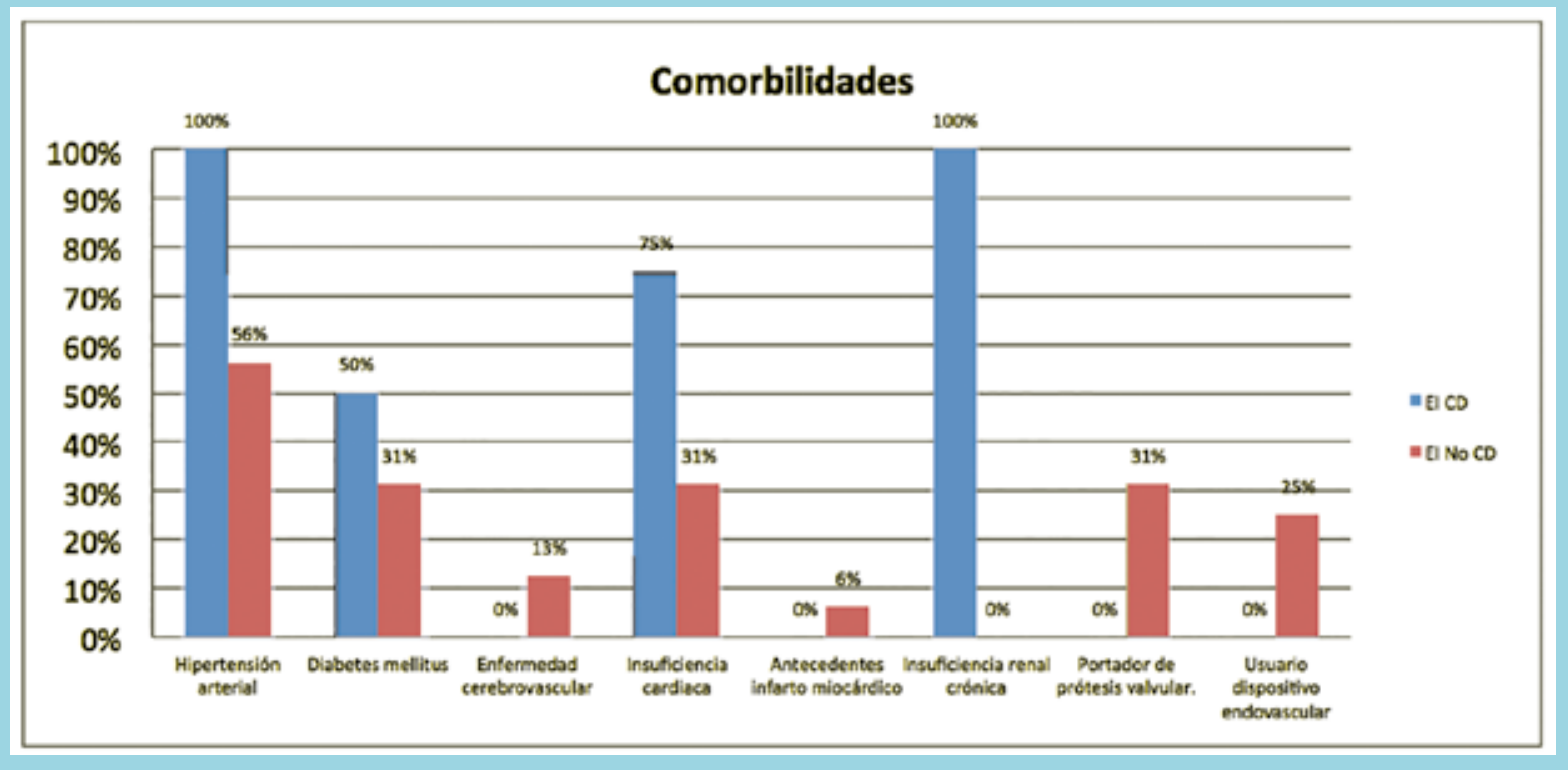




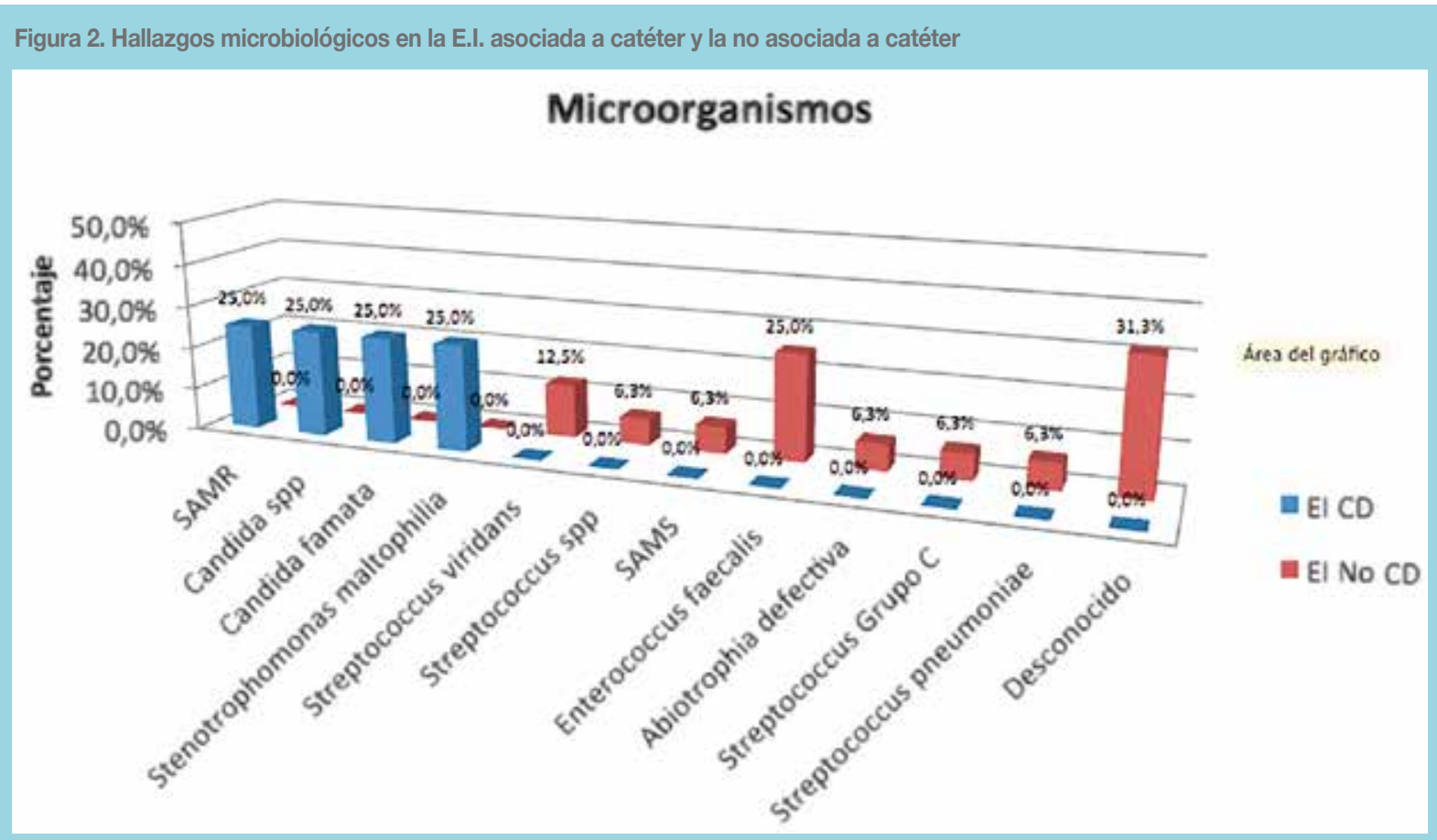

negativos (Figura 2; Tabla 2). Con respecto a los hallazgos ecográficos, en los pacientes con E.I.-CHD la localización más frecuente de las vegetaciones correspondió a la pared de $\mathrm{AD}$, sitio de impacto del jet del CHD en un $100 \%$, seguida por CHD 50\%, tricúspide $25 \%$ y aórtica $25 \%$ (Tablas 3 y 4). Mientras, las válvulas afectadas en pacientes del grupo control corresponden a un $75 \%$ en válvula aórtica y un $25 \%$ en válvula mitral. En sólo un paciente $(6.25 \%)$ se desconoce la zona afectada. Dentro de las complicaciones, en el grupo de E.I.-CHD, la mitad de los pacientes presentó disfunción sistólica e insuficiencia valvular, mientras que en el grupo control fue predominante la insuficiencia valvular $(62,5 \%)$ seguida por la perforación de velo $(25 \%)$.
Imagen 1. ETT eje apical 4 cámaras.

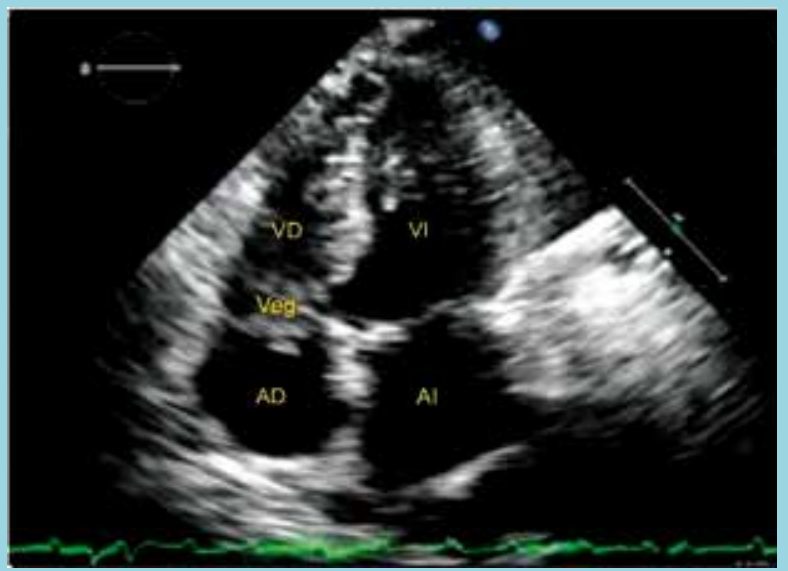

Vegetación por SAMR de gran tamaño en velos tricuspídeos..

Veg: Vegetación; AD: Aurícula derecha; Al: Aurícula izquierda; VI: Ventrículo izquierdo; VD: Ventrículo derecho.
Imagen 2. ETT eje subxifoideo.

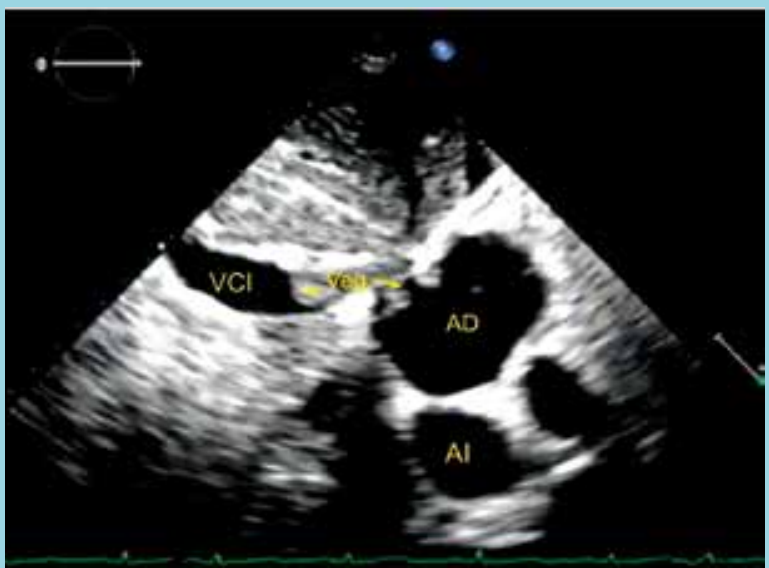

Vegetación por SAMR tanto en desembocadura vena cava inferior como en velos tricuspídeos.

Veg: Vegetación; AD: Aurícula derecha; Al: Aurícula izquierda; VCl: Vena Cava Inferior. 


\section{Discusión:}

La E.I. es una entidad con una mortalidad elevada. Las cifras nacionales muestran una mortalidad de $26.1 \%$ y que, en algunos subgrupos, puede llegar hasta $50 \%{ }^{1}$. Pacientes en hemodiálisis crónica (HDC) tienen un riesgo significativamente mayor para desarrollar E.I. En Estados Unidos la incidencia de E.I. en pacientes en HD es de 308 por 100.000 pacientes al año ${ }^{2}$. Hasta el 31 de Agosto de 2014 en Chile se encontraban 18.160 pacientes en HDC y en la región de Antofagasta la cifra es de 594 pacientes $^{3}$. En Chile se utilizan 3 tipos de accesos: fístulas arteriovenosas (FAV), catéter (transitorio o permanente) y fístula (con prótesis Gorotex o injerto). Del total de pacientes en HD en Chile, $2.720(20,2 \%)$ presentan catéter, de los cuales $484(3,6 \%)$ son transitorios y $2.236(16,6 \%)$ son permanentes ${ }^{3}$. El rango de infección de los catéteres tunelizados es de 14 a $54 \%{ }^{10}$ y tiene unas 6,9 veces mayores probabilidades de muerte por todas las causas y aquellas relacionadas con la infección, en comparación con los pacientes que usan otros accesos. ${ }^{4}$ Un extenso estudio en E.I. asociada a catéter ${ }^{8}$ encontró que los pacientes presentaban alta comorbilidad, pudiendo ocurrir en ausencia de enfermedad valvular. E.I mecanismo sería por un daño al endocardio por presencia del catéter; además, las vías de HD tendrían mayores tasas de infección sistémica. También existiría una asociación entre el desarrollo de E.I. del lado derecho y la presencia de la punta de un catéter cerca o en la AD y en $2 / 3$

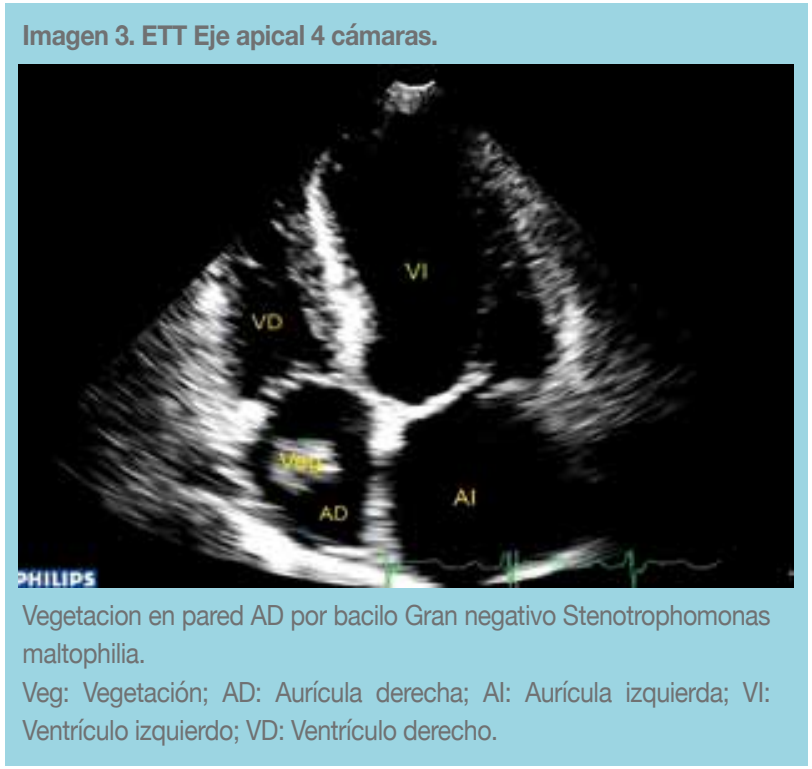

de los casos de E.I asociada a catéter ésta fue del lado derecho ${ }^{8}$. El ETE mostró lesiones que no fueron detectadas por ETT. Los catéteres de HD que requieren un alto flujo idealmente deben ubicarse en la parte superior de la $\mathrm{AD}^{8}$. La endocarditis de cavidades derechas representa entre el 5 y $10 \%$ del total de casos de endocarditis ${ }^{11}$. Situaciones predisponentes son: adicción a drogas por vía parenteral (muy poco frecuente en Chile) y en especial los VIH seropositivos con enfermedad avanzada ${ }^{16}$, portadores de dispositivos intracardiacos, catéteres venosos centrales $^{26}$ (CVC) y cardiopatías congénitas. La válvula

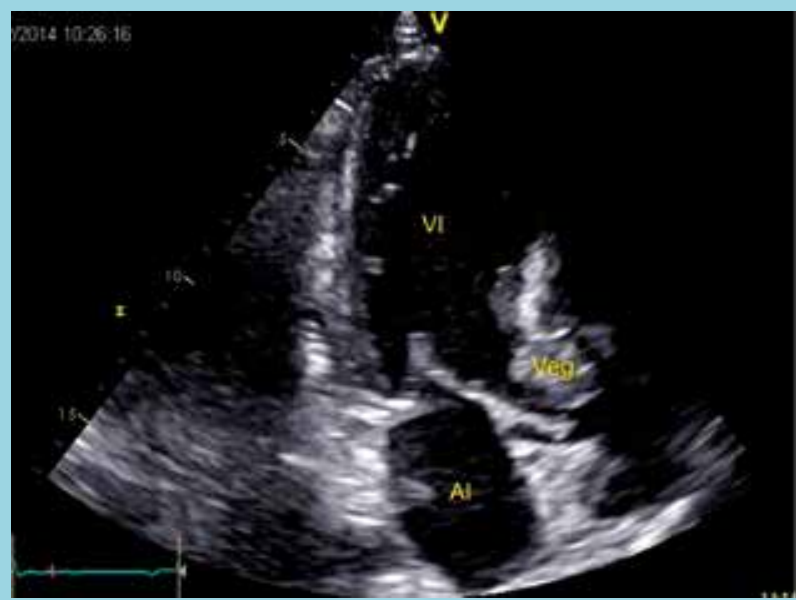

Gran vegetación en velo Aórtico por Cándida Sp. Veg: Vegetación; Al: Aurícula izquierda; Vl: Ventrículo izquierdo.

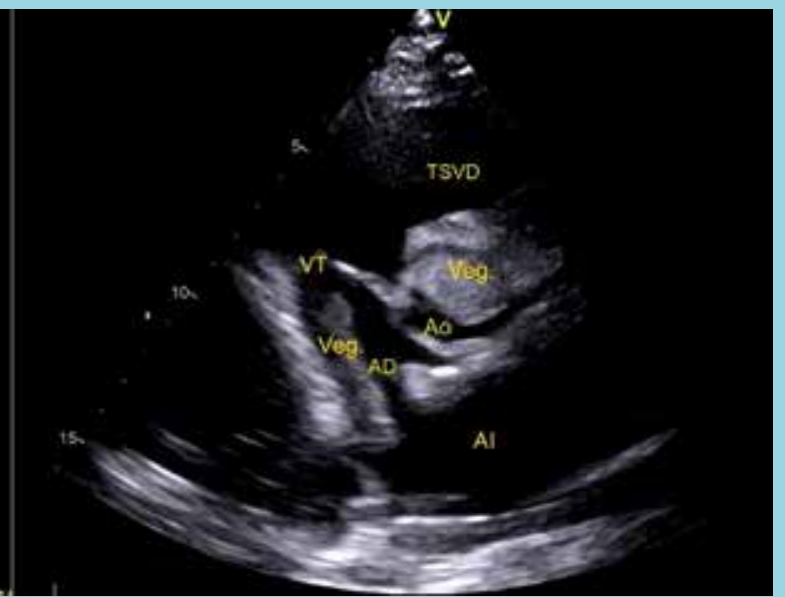

Gran vegetación Aortica y posible gran vegetación pared AD por Candida Sp.

Veg: Vegetación; Al: Aurícula izquierda; AD: Aurícula derecha; Ao: Aorta; TSVD: Tracto Salida Ventrículo Derecho; VT: Valvula Tricuspide. 
Imagen 6A.ETE 2D visión bicava.

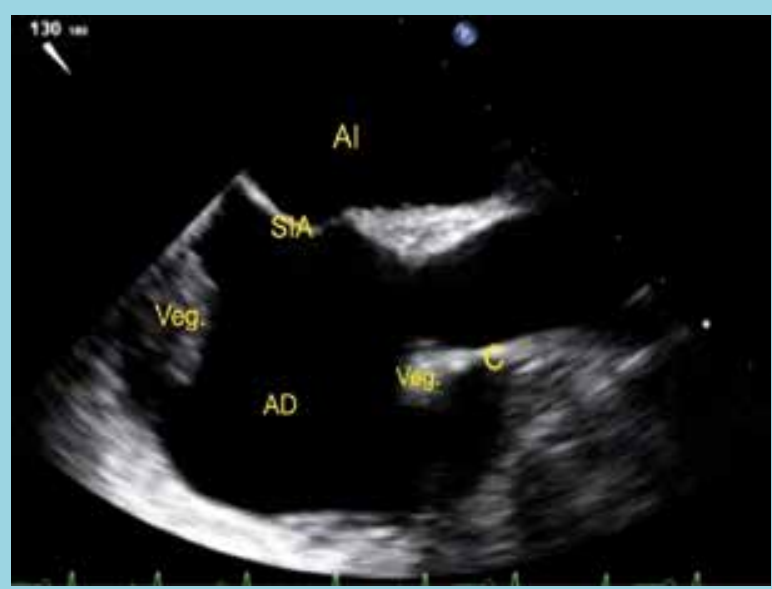

Vegetación por Cándida Famata en punta CHD dentro de la parte superior $A D$ ingresando por VCS, vegetación pared $A D$ cerca desembocadura $\mathrm{VCl}$ en región impacto flujo del jet $\mathrm{CHD}$.

Veg: Vegetación; AD: Aurícula derecha; C: Catéter; VCS: Vena cava superior; SIA: Septum interauricular; Al: Aurícula izquierda; VCl: Vena cava inferior.
Imagen 6B. ETE 3D visión bicava.

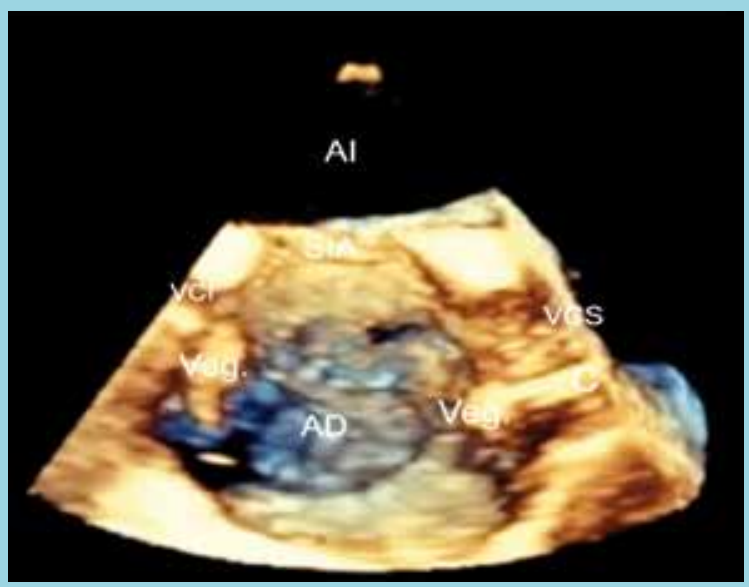

Vegetación por SAMR tanto en desembocadura vena cava inferior como en velos tricuspídeos.

Veg: Vegetación; AD: Aurícula derecha; C: Catéter; VCS: Vena cava superior; Al: Aurícula izquierda; VCl: Vena cava inferior; SIA: Septum interauricular. tricúspide es el lugar preferido de asentamiento, siendo el Staphylococcus aureus el organismo más habitual ${ }^{8-10}$. En Chile, la HD como comorbilidad está presente en el $12,6 \%$ y los catéteres como condición predisponente en el $6,3 \%{ }^{1}$. La mortalidad global en E.I de cavidades derechas es más baja, pero muy variable dependiendo del subgrupo que se trate, fluctuando desde un $4 \%$ hasta un 66\%19-20-21-23-24-25-26. En nuestro estudio, la ETT en todos los casos detectó vegetaciones en la pared de la $\mathrm{AD}$ alrededor de la región de la desembocadura de la VCI. La ETT no permitió aclarar el mecanismo de daño, pero al realizar la ETE 2D y 3D, ésta última permite visualizar en una sola imagen la AD completa y verla de diferentes ángulos y cortes, mostrando que la dirección del jet del catéter de diálisis es directa hacia donde asienta la vegetación, planteándose así que el mecanismo de injuria es por impacto del jet en la pared de la AD provocando el daño del endocardio, trombosis y posterior colonización por el germen. Se insiste en la alta mortalidad entre la asociación de E.I y la presencia de catéter de HD, y en la necesidad de mantener una alta sospecha, pues un manejo precoz pudiese cambiar su evolución. Se debe tener en cuenta que, en este grupo de pacientes, la mortalidad pudo verse influenciada también por ser más añosos, con alta comorbilidad y con infección por microorganismos más agresivos.

\section{Conclusión}

A pesar del escaso número de pacientes, se observó en este grupo que la E.I.-CHD tiene una alta mortalidad, que los gérmenes aislados son poco habituales y asociados a peor evolución. Los hallazgos nos permiten inferir que el mecanismo de daño de los CHD es doble; por un lado, el traumatismo directo del catéter sobre la pared (local) y, por otro, el impacto del flujo del jet sobre la pared de la AD durante la diálisis (a distancia), que en esta serie es la más frecuente. El ETE 2D y 3D, permitió un estudio más exhaustivo, con detección de lesiones no evidenciadas por ETT. 


\section{Referencias}

1. OYONARTE M, MONTAGNA R, BRAUN S, ROJO P, JARA J, CERECEDA M, et al. Endocarditis Infecciosa: Morbimortalidad en Chile. Rev Med Chile 2012; 140: 1517-1528

2. DANIEL J SEXTON. Epidemiology, risk factors and microbiology of infective endocarditis. 2011 UpToDate.

3. BADAL H. XXXIV Cuenta de hemodiálisis crónica (HDC) en Chile. Sociedad de Nefrología de Chile. 2014

4. Allon HYPERLINK "http://www.uptodate.com/contents/tunneled-cuffed hemodialysis-catheter-related-bacteremia/contributors" M HYPERLINK Tunneled, cuffed hemodialysis catheter related bacteremia. 2013 UpToDate

5. BESARAB A, WORK J. 2006 Updates Clinical Practice Guidelines and Recommendations, Vascular Access .National Kidney Foundation, Inc. Work Group Membership. American Journal of Kidney Diseases, 2006; 48: S177.

6. AGUINAGA A, DEL POZO J. Infección asociada a catéter en hemodiálisis: diagnóstico, tratamiento y prevención. NefroPlus 2011;4:1-10

7. ÁLVAREZ M. Accesos vasculares en hemodiálisis. Valdivia, Mayo 2007.

8. CHRISSOHERIS M, LIBERTIN C, RONAN G. ALI, GHANTOUS A, BEKUI A, DONOHUE T. Endocarditis Complicating Central Venous Catheter Bloodstream Infections: A Unique Form of Health Care Associated Endocarditis. Clin. Cardiol. 2009;32:E48-54

9. CASAS R, RIVERO M, CONTRERAS M, MARTÍNEZ A, CRESPO R, MUÑOZ I. Estudio de flujos y eficacia de los catéteres tunelizados durante la hemodiálisis. Servicio de Nefrología. Hospital Universitario RE.I.na Sofía. Córdoba. Comunicaciones Presentadas al XXVIII Congreso Nacional de la SEDEN

10. BEATHARD GA. Management of Bacteremia Associated with Tunneled-Cuffed Hemodialysis Catheters. J Am Soc Nephrol 1999;10: 1045-1049.

11. SHI-MIN YUAN. Right-sided infective endocarditis: recent epidemiologic changes. Int J Clin Exp Med 2014;7:199-218.

12. EVANGELISTA A, GONZALEZ-ALUJAS MT. Echocardio- graphy in infective endocarditis. Heart 2004;90:614-617.

13. TSAI, CHIH-CHAN LIN, CHIH-HSING HSU, WE.I.-TING LI, LI-JEN LIN, et al. Usefulness of real-time three-dimensional echocardiography for diagnosis of infective endocarditis. Scandinavian Cardiovascular Journal, 2009; 43: 318-323.

14. HABIB G, BADANO L, TRIBOUILLOY C, VILACOSTA I, ZAMORANO JL, GALDERISI M, et al. Recommendations for the practice of echocardiography in infective endocarditis European Journal of Echocardiography. 2010;11:202-219.

15. LI JS, SEXTON DJ, MICK N, NETTLES R, FOWLER VG JR, RYAN T, et al. Proposed modification to the Duke criteria for the diagnosis of infective endocarditis. Clin Infect Disease 2000; 30: 633-8.

16. WILSON L, THOMAS D, ASTEMBORSKI J, FREEDMAN T, AND VLAHOV D. Prospective Study of Infective Endocarditis among Injection Drug Users. The Journal of Infectious Diseases 2002;185:1761-6.

17. KORT S, STONY BROOK. Real-time 3-Dimensional Echocardiography for Prosthetic Valve Endocarditis: Initial Experience. J Am Soc Echocardiogr 2006;19:130-139

18. NAQVI T, RAFIE R, GHALICHI M. Real-Time 3D TEE for the Diagnosis of Right-Sided Endocarditis in Patients With Prosthetic Devices. JACC: Cardiovascularimaging, 2010; 3 : 325

19. MARTÍN-DÁVILA P, NAVAS E, FORTUN J, MOYA JL, COBO J, PINTADO V, et al. Analysis of mortality and risk factors associated with native valve endocarditis in drug users: the importance of vegetation size. Am Heart J. 2005;150:1099-106.

20. CACOUB P, LEPRINCE P, NATAF P, HAUSFATER P, DORENT R, WECHSLER B, et al. Pacemaker infective endocarditis.Am J Cardiol 1998;82:480-4.

21. KLUG D, LACROIX D, SAVOYE C, GOULLARD L, GRANDMOUGIN D, HENNEQUIN JL, et al. Systemic infection related to endocarditis on pacemaker leads: clinical presentation and management. Circulation 1997;95:2098-107.

22. NIWA K, NAKAZAWA M, TATENO S, YOSHINAGA M, TERAI M. Infective endocarditis in congenital heart disease: Japanese national collaboration study. Heart. 2005;91:795-800. 
25. YOSHINAGA M, NIWA K, NIWA A, ISHIWADA N, TAKAHASHI H, ECHIGO S, et al. Risk factors for in-hospital mortality duringinfective endocarditis in patients with congenital heart disease. Am J Cardiol. 2008;101:114-8.

24. LI W, SOMERVILLE J. Infective endocarditis in the grownup congenital heart $(\mathrm{GUCH})$ population. Eur Heart J. 1998;19:166-73.

26. MANCINI L, GODOY G, MUJICA C, KOCIUBINSKY P, POTITO M, CORSIGLIA D, et al. Epidemiología de la endocarditis infecciosa derecha. Rev Fed Arg Cardiol 2002; 31: 309-31 